\title{
Upright Positive Expiratory Pressure Therapy and Exercise: Effects on Gastroesophageal Reflux in COPD and Bronchiectasis
}

\author{
Annemarie L Lee PhD, Linda Denehy PhD, John W Wilson PhD, Stuart Roberts MD, \\ Robert G Stirling MD, Ralf G Heine MD, and Brenda M Button PhD
}

\begin{abstract}
BACKGROUND: Patients with COPD and patients with bronchiectasis undertake airway clearance therapy and exercise as part of management, but the effect of these activities on gastroesophageal acid exposure is unknown. This study aimed to determine if positive expiratory pressure (PEP) therapy and standardized exercise tasks were associated with increased gastroesophageal reflux. METHODS: During dual-probe 24 hour esophageal pH monitoring, all participants undertook a single session of PEP therapy, a measure of submaximal exercise capacity (6-min walk test [6MWT]), and a functional upper limb task (grocery shelving task [GST]). The number of reflux episodes and fractional reflux time (reflux index [RI]) were recorded during each intervention and compared to equivalent background time (BGT). RESULTS: Fifty-seven participants (30 with bronchiectasis, 27 with COPD, mean \pm SD age $61 \pm 13$ y, FEV $_{1} 61.2 \pm 24.6 \%$ predicted) completed the study. Episodes of isolated distal esophageal reflux occurred in $30 \%$ of participants during PEP therapy, 22\% during the 6MWT, and $20 \%$ during the GST. However, there was no significant difference in distal RI during 6MWT or PEP therapy, compared to BGT (all $P>.05$ ). The number of reflux episodes was decreased, compared to BGT during the GST $(P=.001)$ and $6 \mathrm{MWT}$ $(P=.001)$, but not during PEP therapy $(P=.71)$. CONCLUSIONS: Episodes of gastroesophageal reflux may occur during physiotherapy tasks, including airway clearance therapy using mouthpiece PEP, the 6MWT, and a measure of upper limb movement. However, as these activities did not increase the frequency of these events, no modifications to these tasks to minimize the occurrence of gastroesophageal reflux are necessary. Key words: COPD; bronchiectasis; gastroesophageal reflux; exercise; exercise tests; respiratory therapy. [Respir Care 2012;57(9):1460-1467. ( 2012 Daedalus Enterprises]
\end{abstract}

\section{Introduction}

Chronic cough, sputum production, and exercise intolerance secondary to exertional dyspnea are common fea-

Drs Lee and Denehy are affiliated with the Department of Physiotherapy, Melbourne School of Health Sciences; and Drs Wilson and Heine are affiliated with the Department of Medicine, The University of Melbourne, Melbourne, Australia. Drs Lee and Button are affiliated with the Department of Physiotherapy; Drs Wilson and Stirling are affiliated with the Department of Allergy, Immunology, and Respiratory Medicine; and Dr Roberts is affiliated with the Department of Gastroenterology, Alfred Hospital, Melbourne, Australia. Drs Wilson, Roberts, Stirling, and Button are also affiliated with the Department of Medicine, Monash University, Clayton, Australia. Dr Heine is also affiliated with the Department of Gastroenterology and Clinical Nutrition, Royal Children's Hospital, Melbourne, Australia.

This research was partly supported by the Physiotherapy Research Foundation of the Australian Physiotherapy Association, and the Australian National Health and Medical Research Council. tures of COPD and bronchiectasis. ${ }^{1,2}$ This clinical profile lends support to the prescription of airway clearance therapy for treatment of excess secretions and physical exercise in both conditions. ${ }^{3-5}$ Increased gastroesophageal reflux (GER), diagnosed with 24 hour esophageal $\mathrm{pH}$

\footnotetext{
Dr Lee presented a version of this paper at the meeting of the Thoracic Society of Australia and New Zealand, held March 30-April 2, 2008, in Melbourne, Australia, and at the National Cardiorespiratory Group Conference of the Australian Physiotherapy Association, held October 1-5, 2009, in Sydney, Australia.
}

The authors have disclosed no conflicts of interest.

Correspondence: Annemarie L Lee PhD, Department of Physiotherapy, University of Melbourne, 200 Berkeley Street, Melbourne, Victoria 3004, Australia. E-mail: annlee@unimelbeduau.

DOI: $10.4187 /$ respcare. 01498 
monitoring, has been identified in COPD and bronchiectasis. $^{6-9}$ Various physiological mechanisms, including lower esophageal sphincter relaxations and a raised intraabdominal pressure, have the potential to increase GER events $^{10-13}$ and these may arise during specific exercise tasks or airway clearance therapies (including cough) ${ }^{14}$ prescribed by a physiotherapist. To determine whether modifications to these techniques are necessary to minimize GER in COPD and bronchiectasis, it is important to explore their effect on gastroesophageal acid exposure.

With the exception of standard gravity-assisted drainage in patients with cystic fibrosis, ${ }^{15-17}$ the impact of airway clearance techniques on gastroesophageal acid exposure is not well defined. Positive expiratory pressure (PEP) therapy is a technique involving resisted expiration through a mask or mouthpiece, forced expirations, and coughing, ${ }^{18}$ often performed in an upright, seated position. One study demonstrated fewer reflux symptoms during PEP therapy, compared to gravity-assisted drainage in 6 adolescents, and this was attributed to the upright posture throughout the technique. ${ }^{16}$ However, as esophageal $\mathrm{pH}$ monitoring was not concurrently applied, it is not known whether PEP therapy with forced expirations and coughing induce or exacerbate GER. PEP is often prescribed in clinical practice as part of physiotherapy management of COPD and bronchiectasis, ${ }^{19}$ but no study has explored its effects on the frequency and duration of proximal and distal GER episodes, as measured by 24 hour esophageal $\mathrm{pH}$ monitoring in these respiratory conditions.

Increased GER has been demonstrated during steady pace walking, upper limb training, and tasks incorporating trunk flexion in individuals without respiratory disease, and is attributed to exercise-induced reductions in lower esophageal sphincter pressure or raised intra-abdominal pressure. ${ }^{11,20,21}$ Physical exercise incorporating lower and upper limb function is frequently applied in patients with COPD and patients with bronchiectasis, with the intensity based on standardized measures of submaximal endurance

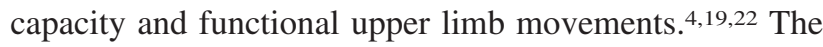
impact of these exercise activities on GER events in these patient populations is unknown.

Therefore, the aim of this study was to determine if airway clearance therapy using PEP therapy in an upright, seated position, a submaximal exercise test, and a functional upper limb task increase the incidence of GER episodes, measured by dual-channel 24 hour esophageal $\mathrm{pH}$ monitoring in patients with COPD or bronchiectasis.

\section{Methods}

\section{Subjects}

The medical records of patients with COPD or bronchiectasis who attended respiratory out-patient clinics of the

\section{QUICK LOOK}

\section{Current knowledge}

Airway clearance maneuvers are commonly performed in patients with COPD, and, along with exercise, are important components of a pulmonary rehabilitation program. Gastroesophageal reflux is a common comorbidity in these patients.

\section{What this paper contributes to our knowledge}

Airway clearance using positive expiratory pressure via mouthpiece, performance of the 6-min walk test, and measures of upper limb movement are not associated with an increase in the number of episodes of gastroesophageal reflux.

Alfred Hospital, Melbourne, Victoria, Australia, a tertiary institution, between September 2005 and May 2007, were screened for inclusion. A diagnosis of COPD was confirmed according to the Global Initiative for Chronic Obstructive Lung Disease (GOLD) criteria (stages I to IV), ${ }^{23}$ while bronchiectasis was confirmed by high-resolution computed tomography. ${ }^{24}$ Patients who met the eligibility criteria and agreed to undertake a prevalence study of GER were included. Exclusion criteria were a diagnosis of both COPD and bronchiectasis, a known history of asthma, cystic fibrosis, interstitial lung disease, or known esophageal varices or strictures. Those prescribed anti-reflux medication (histamine type-2 receptor antagonists, prokinetic medications, and proton pump inhibitors) ceased their medication for 7 days prior to the study, while antacids were withheld the day before and during the study. All participants gave informed consent, and our human research ethics committee approved the study.

\section{Protocol}

Ambulatory dual-channel 24 hour esophageal $\mathrm{pH}$ monitoring (Polygram 98 ambulatory $\mathrm{pH}$ testing system with Digitrapper, Medtronic, Minneapolis, Minnesota) was used to measure GER. A dual-channel $\mathrm{pH}$ catheter with 2 monocrystalline antimony electrodes spaced $15 \mathrm{~cm}$ apart (Zinetics, Sweden, and Synectics, Portugal) was positioned with the distal $\mathrm{pH}$ sensor $5 \mathrm{~cm}$ above the lower esophageal sphincter, with the proximal sensor located $15 \mathrm{~cm}$ above the distal sensor. ${ }^{25}$ The catheter was connected to a portable digital recorder (Digitrapper), storing $\mathrm{pH}$ data for a 24 hour period. During the study, all participants recorded meal times, time spent in erect and supine positions, and symptoms of GER. During the 24 hours, 3 interventions were completed in the following order: a single session of 
PEP therapy on day 1, followed by two 6-min walk tests (6MWT), and a measure of function involving upper limb movement, the grocery shelving task (GST) on day 2. All were completed at an interval of $>2$ hours after meals. Participants were instructed to undertake a normal day with respect to oral intake, recumbent positioning, and other daily activities to ensure an accurate reflection of GER events over the 24 hour study. No restrictions with regard to type and quantity of food or drink or time spent in recumbent or upright positions were imposed, although participants were instructed to record the type of food and drink consumed, to determine the association between a diagnosis of GER and specific oral intake. ${ }^{26}$

\section{PEP Therapy}

During the first afternoon of $\mathrm{pH}$ monitoring, a session of PEP therapy using mouthpiece PEP (PariPEP, Pari Respiratory Equipment, Midlothian, Virginia) with a nose clip was completed. This device consists of a mouthpiece and one way valve and a series of resistors ranging from 1.5 to $5 \mathrm{~mm}$ at the expiratory orifice. A manometer determining the correct level of PEP was included within the circuit between the valve and the resistor. The diameter of the resistor used to give a steady PEP of $10-20 \mathrm{~cm} \mathrm{H}_{2} \mathrm{O}$ during the middle part of expiration was determined for each participant. ${ }^{18}$ This pressure was maintained during tidal volume breathing with slightly active expirations against the resistor. Treatment was undertaken in an upright, with elbows supported, seated position with a noseclip in place and lips sealed around the mouthpiece. A session consisted of periods of breathing with PEP for 10 breaths, followed by the forced expiratory technique and coughing. The forced expiratory technique consisted of a combination of 1 or 2 forced expirations (huffs) from low to high lung volumes, to assist in sputum expectoration, followed by a period of breathing control, then coughing (up to 3 coughs) to clear secretions. ${ }^{18}$ A total of 8 cycles of PEP breathing, forced expiratory technique, and coughing were completed for each participant, with the total number of coughs recorded.

\section{6-Min Walk Test}

Each participant completed 2 6MWTs on the second day of monitoring, with one hour separating each test. The 6MWT was completed indoors on a flat, enclosed corridor of $30 \mathrm{~m} .{ }^{27}$ Each participant was instructed to walk, attempting to cover as much ground as possible within $6 \mathrm{~min}$, with standardized verbal encouragement. $\mathrm{S}_{\mathrm{pO}_{2}}$ was monitored throughout the test, using a pulse oximeter (MagnaTek 33, ProACT Medical Systems, Frenchs Forest, New South Wales, Australia). Before and after the test, data were obtained for $\mathrm{S}_{\mathrm{pO}_{2}}$, heart rate, respiratory rate, Borg scale dyspnea score, ${ }^{28}$ and blood pressure. The 6MWT distance was measured and the start and finish time for each 6MWT was recorded.

\section{Grocery Shelving Task}

Each participant completed the GST on the second day of monitoring, according to the prescribed protocol. ${ }^{22}$ This test required that 20 grocery cans, each weighing $420 \mathrm{~g}$, contained in 2 shopping bags (each bag containing 10 cans), were positioned onto a shelf within an open cupboard at approximately $15 \mathrm{~cm}$ above the shoulder height of the patient. The bags were first retrieved from the floor and positioned onto a trolley that was $90 \mathrm{~cm}$ from the floor. Each participant then positioned the cans onto the shelf as quickly as possible. Participants were instructed to pace themselves and slow down or rest if required. The time taken to complete the task was measured in seconds. Baseline physiological measures (heart rate, $\mathrm{S}_{\mathrm{pO}_{2}}$, respiratory rate), and the Borg scale for perceived breathlessness $^{28}$ were recorded at rest and repeated immediately on completion of the task. Following a practice test, 3 repeated trials of the GST were completed, and the time taken to perform the GST was recorded in seconds. ${ }^{22}$ The task completed in the shortest duration was recorded. The overall time to complete the protocol for the GST from the practice test to the conclusion of 3 tasks was recorded and expressed as the total time for the GST.

After the 24 hour period of monitoring, data were downloaded into a computer file (Polygram $98 \mathrm{pH}$ testing application software, Medtronic, Minneapolis, Minnesota) for analysis of proximal and distal GER. A diagnosis of increased GER was based on a DeMeester score exceeding $14.72^{29}$ and/or proximal esophageal acid exposure time (reflux index $[\mathrm{RI}])>0.9 \% .^{30}$ The number of reflux episodes per minute (NRE) and fraction of reflux time (RI) in the proximal and distal esophagus per minute were the principal GER parameters measured during each intervention (PEP therapy, 6MWT, and GST). These parameters were compared to GER parameters during the "background reflux time" (BGT). BGT was defined as the period of time over 24 hours, which excluded meals and postprandial period, time spent in a recumbent position, and time occupied by physiotherapy interventions. The proportion of NRE was expressed as episodes per minute, to correct for the different duration between interventions and BGT for a comparable evaluation of GER parameters.

\section{Data Management}

In a pilot study of 14 patients, $14 \%$ demonstrated increased episodes of GER during PEP therapy, compared to BGT. Based on these data, a total of 57 patients were required to achieve statistical power of $80 \%$ with a signif- 


\section{UpRIght PEP AND EXERCISE FOR REFLUX IN COPD AND BRONCHIECTASIS}

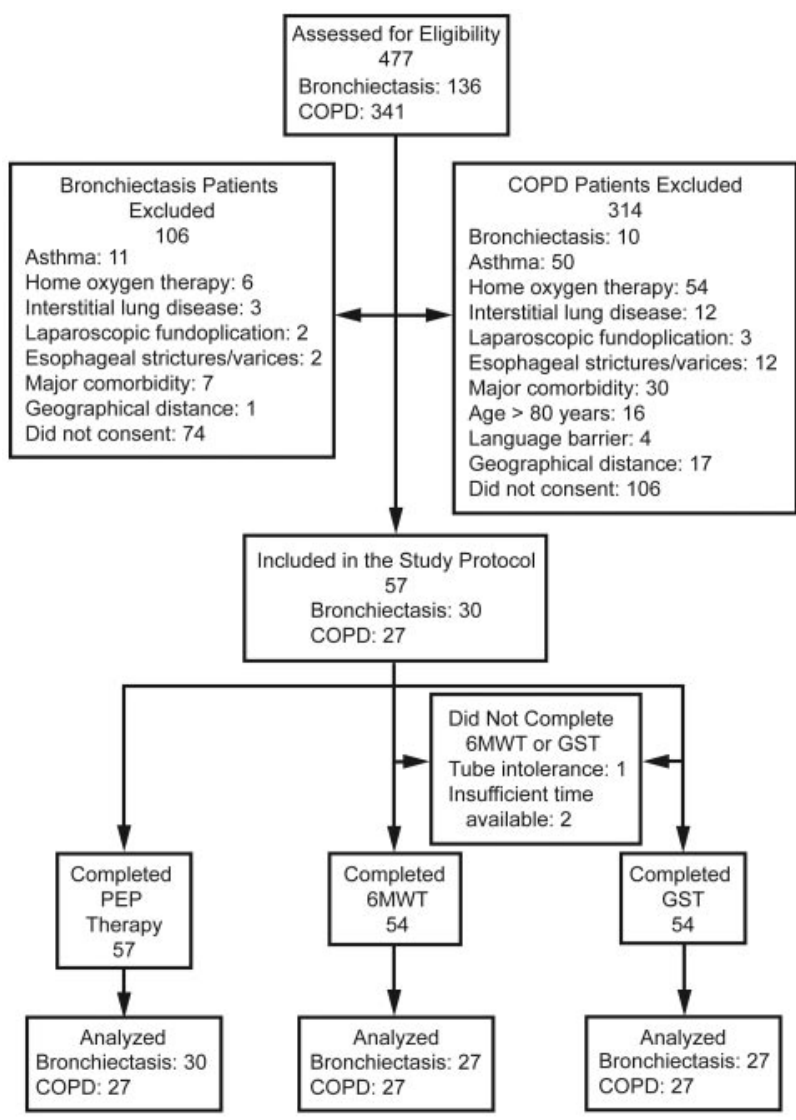

Fig. 1. Flow of study participants. PEP = positive expiratory pressure. $6 \mathrm{MWT}=6$-min walk test. GST $=$ grocery shelving task.

icance level of $<.05$. Data are described using mean $\pm \mathrm{SD}$, or median and interquartile range for non-parametric data. All GER data were not normally distributed and could not be adequately transformed; therefore, non-parametric analysis was completed. Comparison of NRE and RI between each intervention and BGT was analyzed using the Wilcoxon signed rank test. The relationships between GER parameters and 6MWT distance, GST time, and number of coughs during PEP therapy were analyzed using the Pearson product moment. Alpha was set at .05 , with adjustments for multiple comparisons using the Bonferroni correction.

\section{Results}

A total of 341 patients with COPD and 136 with bronchiectasis were screened for eligibility, with 180 meeting the inclusion criteria. The flow of participants through the study and reasons for exclusion are illustrated in Figure 1. Overall, 57 participants with chronic respiratory disease (30 with bronchiectasis, and 27 with COPD) with a mean $\pm \mathrm{SD}$ age of $61 \pm 13$ years and a mean $\mathrm{FEV}_{1}$ of $61.2 \pm 24.6$ percent of predicted were included, with de-
Table 1. Participant Demographics $(n=57)$

\begin{tabular}{|c|c|c|}
\hline & COPD & Bronchiectasis \\
\hline Male/female, no. & $21 / 6$ & $13 / 17$ \\
\hline Age, y & $67.7 \pm 7.7$ & $53.7 \pm 14.0$ \\
\hline Body mass index, $\mathrm{kg} / \mathrm{m}^{2}$ & $26.9 \pm 4.7$ & $26.6 \pm 5.8$ \\
\hline $\mathrm{FEV}_{1}, \%$ predicted & $47.2 \pm 17.4$ & $73.9 \pm 23.4$ \\
\hline $\mathrm{FVC}, \%$ predicted & $87.7 \pm 24.5$ & $89.4 \pm 20.2$ \\
\hline $\begin{array}{l}\text { Prescribed anti-reflux medication, } \\
\text { no. }(\%)^{*}\end{array}$ & $17(63)$ & $16(53)$ \\
\hline Antacids, no. (\%) & $7(25)$ & $12(40)$ \\
\hline Histamine $_{2}$ antagonists, no. (\%) & $2(7)$ & $3(10)$ \\
\hline Proton pump inhibitors, no. (\%) & $12(43)$ & $14(46)$ \\
\hline
\end{tabular}
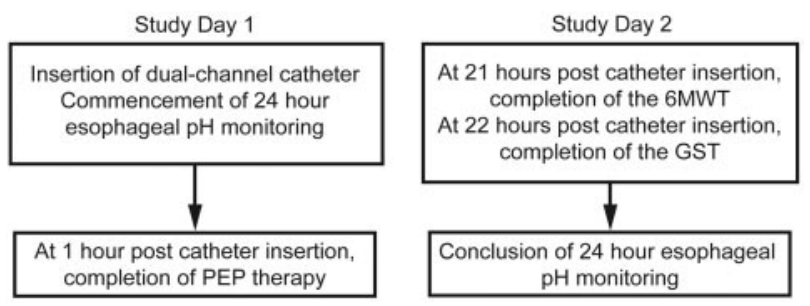

Fig. 2. Timing of physiotherapy tasks over the 24 hour period. $6 \mathrm{MWT}=6$-min walk test. GST $=$ grocery shelving task. PEP $=$ positive expiratory pressure.

mographic characteristics outlined on Table 1. All 57 completed PEP therapy, while 54 participants completed the 6MWT and the GST. Two individuals arrived too late on the second day of monitoring for testing to be completed, while one was unable to tolerate the $\mathrm{pH}$ monitoring for the complete 24 hours, hence it was removed prior to completion of the exercise tests. All 3 participants who did not perform exercise testing had bronchiectasis.

The timing of each physiotherapy task over the 24 hours is outlined in Figure 2. The mean \pm SD 6MWT distance was $495 \pm 125.67 \mathrm{~m}$, while the time required to complete the GST overall was $36.2 \pm 8.4 \mathrm{~s}$. The total time for the PEP therapy session ranged between 21 to $35 \mathrm{~min}$, with an average of $21 \pm 7$ coughs per session. A total of 10 participants with COPD (37\%) and 12 with bronchiectasis (40\%) met the diagnostic criteria for increased GER. ${ }^{29,30}$

In COPD there was no difference in the overall oral intake during the 24 hours in those diagnosed with GER, compared to those without GER $(P=.68)$. A total of 6 participants with COPD had a diet that included caffeine $(n=6)$, high fat content $(n=6)$, chocolate $(n=3)$, and/or alcohol $(n=3)$. This dietary intake was not associated with a diagnosis of GER. Similarly, there was no difference in the overall oral intake during the 24 hours in those diagnosed with GER, compared to those without GER, in 
bronchiectasis $(P=.49)$. Only 5 participants with bronchiectasis had a diet over the 24 hour period that included caffeine $(n=3)$ and/or a high fat content meal $(n=2)$, with no participants consuming chocolate or alcohol during the study. This diet was not associated with a diagnosis of GER.

There was no difference in the number of participants with COPD diagnosed with GER who were prescribed anti-reflux medication, versus treatment naïve participants $(P=.08)$, nor was there a difference in those with bronchiectasis $(P=.46)$. There was no difference in the proportion of participants who experienced reflux during both baseline and exercise interventions and who were prescribed anti-reflux medication, compared to treatment naïve participants in COPD (66\% vs $33 \%, P=.52)$ or in bronchiectasis (60\% vs $40 \%, P=.13)$. Similarly, there was no difference in the proportion of participants who experienced reflux during both baseline and airway clearance therapy intervention and who were prescribed antireflux medication compared to treatment naïve participants in COPD (66\% vs $33 \%, P=.19)$ or in bronchiectasis (63\% vs $36 \%, P=.44)$.

Overall 31 participants (54\%, 17 with bronchiectasis) experienced GER episodes during at least one physiotherapy task, with 7 (28\%) experiencing reflux episodes during all interventions. Figure 3 depicts the number of participants with COPD and participants with bronchiectasis who experienced distal only reflux events or distal and proximal reflux events during each task.

There was no significant difference in the NRE or RI in the distal esophagus during PEP therapy, compared to BGT (Table 2). When analyzed as a subgroup, there were fewer NRE and RI in the distal esophagus, compared to BGT, in those with COPD $(P<.001)$, but not bronchiectasis. Both conditions experienced a lower NRE in the proximal esophagus (both $P<.001$ ) compared to BGT.

During the 6MWT and GST the proximal and distal NRE and proximal RI were significantly less during the task, compared to BGT, with no difference in distal RI (Tables 3 and 4). In COPD, there were fewer distal NRE $(P<.001)$ and a lower distal RI $(P=.003)$ during the 6MWT, compared to BGT, but no difference during the GST. In bronchiectasis the distal and proximal NRE and RI were similar during the GST and 6MWT, compared to BGT. Irrespective of a diagnosis of GER, ${ }^{29,30}$ there were significantly fewer distal GER episodes during PEP therapy, the 6MWT, and the GST, compared to BGT in COPD and bronchiectasis.

As only 2 participants experienced episodes of reflux in the proximal esophagus during any intervention, the relationship between the 6MWT distance, the time required to complete the GST, and the number of coughs during PEP therapy were related to distal GER indices only. There was no relationship between the distance achieved in the 6MWT,

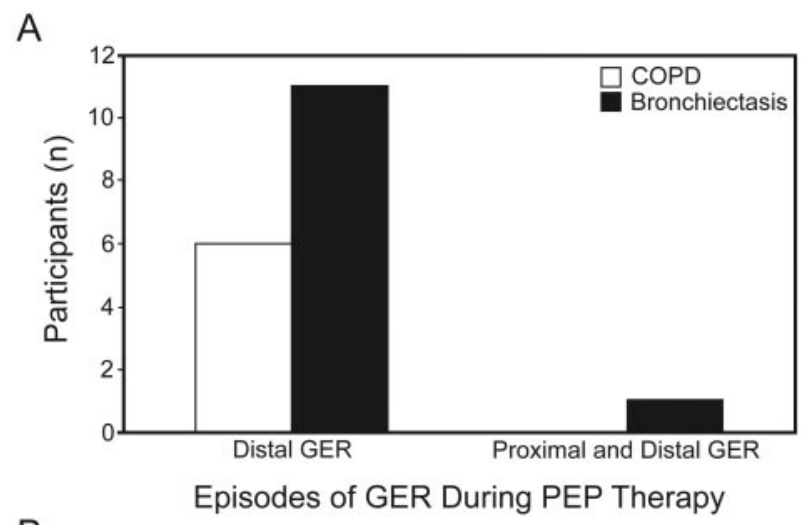

B
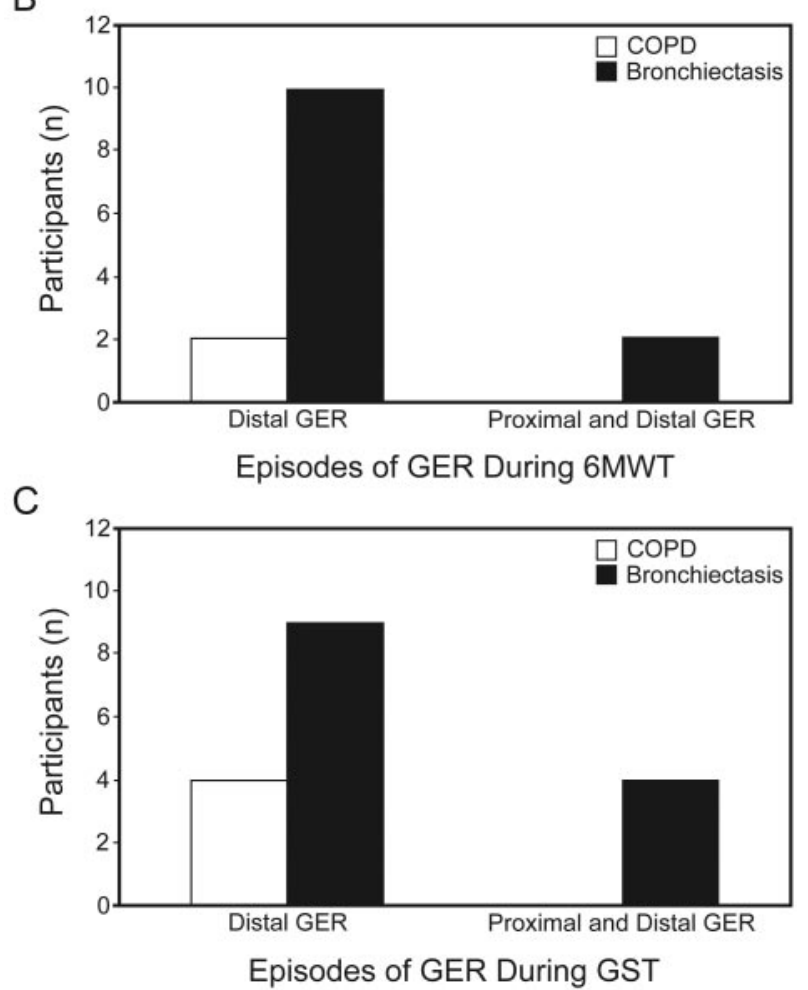

Fig. 3. Number of participants with COPD and bronchiectasis who experienced distal only gastroesophageal reflux (GER) or distal and proximal GER during (A) positive expiratory pressure (PEP) therapy, (B) 6-min walk test, and (C) grocery shelving task (GST).

time taken to complete the GST, or number of coughs recorded during the overall PEP therapy session and the distal NRE or RI (all $\mathrm{r}<0.25, P>.05$ ).

\section{Discussion}

To our knowledge, this is the first study examining the effect of PEP therapy and exercise tests on proximal and distal GER events in COPD or bronchiectasis. While a proportion of participants (54\%) experienced GER during at least one physiotherapy task, the frequency of these events during the 6MWT and GST were less, compared to 
Table 2. Comparison of GER Parameters Between PEP Therapy and Background Time

\begin{tabular}{lccc}
\hline \hline \multicolumn{1}{c}{ GER Measure } & $\begin{array}{c}\text { Background Time } \\
\text { Median (IQR) }\end{array}$ & $\begin{array}{c}\text { PEP Therapy } \\
\text { Median (IQR) }\end{array}$ & $P^{*}$ \\
\hline Distal NRE/min & $0.08(0.04-0.13)$ & $0.00(0.00-1.00)$ & .71 \\
Distal RI/min & $0.00(0.00-0.01)$ & $0.00(0.00-0.25)$ & .64 \\
Proximal NRE/min & $0.02(0.00-0.03)$ & $0.00(0.00-0.00)$ & $<.001$ \\
Proximal RI/min & $0.00(0.00-0.00)$ & $0.00(0.00-0.00)$ & .83
\end{tabular}

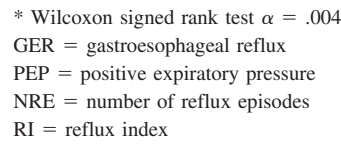

Table 3. Comparison of GER Parameters Between 6MWT and Background Time

\begin{tabular}{lccc}
\hline \hline \multicolumn{1}{c}{ GER Measure } & $\begin{array}{c}\text { Background Time } \\
\text { Median (IQR) }\end{array}$ & $\begin{array}{c}\text { 6MWT } \\
\text { Median (IQR) }\end{array}$ & $P^{*}$ \\
\hline Distal NRE/min & $1.62(0.68-2.44)$ & $0.00(0.00-1.00)$ & $<.001$ \\
Distal RI/min & $0.07(0.02-0.13)$ & $0.00(0.00-0.10)$ & .36 \\
Proximal NRE/min & $0.30(0.10-0.65)$ & $0.00(0.00-0.00)$ & $<.001$ \\
Proximal RI/min & $0.01(0.00-0.02)$ & $0.00(0.00-0.00)$ & $<.001$
\end{tabular}

\footnotetext{
* Wilcoxon signed rank test $\alpha=.004$

GER $=$ gastroesophageal reflux

$6 \mathrm{MWT}=6$-min walk test

$\mathrm{NRE}=$ number of reflux episodes

$\mathrm{RI}=$ reflux index
}

Table 4. Comparison of GER Parameters Between GST and Background Time

\begin{tabular}{lccc}
\hline \hline \multicolumn{1}{c}{ GER Measure } & $\begin{array}{c}\text { Background Time } \\
\text { Median (IQR) }\end{array}$ & $\begin{array}{c}\text { GST } \\
\text { Median (IQR) }\end{array}$ & $P^{*}$ \\
\hline $\begin{array}{l}\text { Distal NRE/min } \\
\text { Distal RI/min }\end{array}$ & $0.93(0.41-1.53)$ & $0.00(0.00-0.25)$ & $<.001$ \\
Proximal NRE/min & $0.18(0.06-0.41)$ & $0.00(0.00-0.00)$ & $<.001$ \\
Proximal RI/min & $0.00(0.00-0.02)$ & $0.00(0.00-0.00)$ & $<.001$ \\
& & & \\
* Wilcoxon signed rank test $\alpha=.004$ & & \\
GER $=$ gastroesophageal reflux \\
GST $=$ grocery shelving task \\
NRE $=$ number of reflux episodes \\
RI $=$ reflux index
\end{tabular}

BGT. Similarly, a session of PEP therapy was not associated with a significant increase in reflux episodes in the proximal and distal esophagus. The occurrence of reflux episodes during each activity was unrelated to the overall duration of each exercise task or coughing frequency during PEP therapy.

The lack of an increase in reflux episodes during PEP therapy, compared to BGT, may be attributable to several factors. It may be related to the position during which the airway clearance technique was completed. Although reflux episodes generally occur in greater frequency in an upright position in individuals without lung disease, events are generally of a short duration. ${ }^{12,31}$ It may be that the upright position in which PEP therapy was completed is central to limiting distal and proximal GER in COPD and bronchiectasis, ${ }^{16}$ an observation that has been previously considered, using a comparable protocol of PEP therapy in cystic fibrosis. ${ }^{16}$ This may account for the small number of participants who experienced GER events during PEP therapy in the current study.

Self-perpetuating cycles of coughing are associated with a higher prevalence of GER in individuals diagnosed with chronic cough. ${ }^{32}$ While cycles of inspiration and expiration through the PEP device were interspersed with forced expiratory maneuvers and coughing in the current study, this practice was well controlled, and paroxysmal uncontrolled coughing was avoided. The lack of relationship between the number of coughs and reflux events in the proximal and distal esophagus implies that the frequency of coughing in the current study did not increase the occurrence of GER episodes in bronchiectasis and COPD during PEP therapy.

The importance of the respiratory cycle in the few reflux episodes detected during PEP therapy is unclear. Previous physiological studies reported a rise in intra-abdominal pressure during inspiration and expiration, ${ }^{33}$ and our participants were instructed to inspire to a moderate depth and expire with pressures of $10-20 \mathrm{~cm} \mathrm{H}_{2} \mathrm{O}$. It is possible that any change in intra-abdominal pressure during this respiratory pattern may have been insufficient to trigger increased GER events. In addition, increased activity of the crural diaphragm during straining or coughing has been documented, a response that serves to augment the lower esophageal sphincter pressure and minimize reflux. ${ }^{13,34} \mathrm{Al}-$ ternatively, the presence of raised intra-abdominal pressure may be important only if accompanied by a low basal lower esophageal sphincter pressure, ${ }^{13,35}$ a condition that may be absent in the current sample of participants.

There are several reasons for the lack of increased GER events during the 6MWT. First, meeting the diagnostic criteria for increased GER (DeMeester score $>14.72$ and/or proximal RI $>0.9 \%)^{29,30}$ may be important. Previous studies demonstrated that both steady paced and brisk treadmill walking were associated with increased reflux episodes in individuals with confirmed GER, ${ }^{20}$ but not in healthy individuals without GER. ${ }^{21}$ The small proportion of participants (10 with COPD, 12 with bronchiectasis) diagnosed with increased GER in the current study may account for the reduced frequency of reflux episodes during the 6MWT, compared to BGT.

The intensity of exercise may also be important. ${ }^{11}$ High intensity tasks such as running or jogging at up to $85 \%$ of maximal heart rate are associated with increased GER, when compared to $\mathrm{BGT}^{11,21}$ and are attributable to the rise in intra-abdominal pressure in response to the increased 
expiration. ${ }^{36,37}$ While the 6MWT has demonstrated a strong relationship between measures of maximal exercise performance in patients with COPD and bronchiectasis, ${ }^{38,39}$ our protocol for the test ${ }^{27}$ prohibited participants from running during the task. The consistency of pace during walking may also be important. Ravi et $\mathrm{al}^{20}$ found treadmill based walking at $1 \mathrm{~km} / \mathrm{h}$ and $4.2 \mathrm{~km} / \mathrm{h}$ associated with reflux events, although no analysis of BGT versus exercise time was undertaken, making comparisons with the current study limited. Despite this limitation, the need to maintain a continual speed on a treadmill, even at a slow rate, may have contributed to the raised frequency of reflux events. ${ }^{20}$ In contrast, participants in the current study were permitted to vary the pace during the 6MWT, according to their physical limitations. While the intensity of the 6MWT was likely to be limited by respiratory symptoms in some patients, the instructions for the test included directions to "cover as much distance as possible in 6 minutes." 27 These results lend some support to the hypothesis that this formal measure of exercise capacity, even at a brisk pace, did not increase the risk of reflux events in COPD or bronchiectasis.

The effect of upper limb exercises on GER events has been primarily examined during weight lifting tasks, including bench press and seated arm press activities. ${ }^{36}$ With a greater intensity and different starting positions (reclined and seated), these tasks are unlikely to simulate the movements involved in the GST, making comparisons difficult. The lack of difference in distal and proximal GER parameters, compared to BGT, during the GST, may be related to the test protocol. It is principally a measure of upper limb function, and participants were instructed to complete the task in the shortest possible time. ${ }^{22}$ Although the task included trunk flexion, participants were advised to pace themselves and could slow down and rest if required. ${ }^{22}$ The lack of relationship between the total time for the GST and the distal GER parameters indicates that the intensity of this activity had minimal influence on gastroesophageal acid exposure.

The few GER events during both the 6MWT and the GST may also be related to the short time frame in which these tasks were completed. Each 6MWT was performed in 6 minutes (12 $\mathrm{min}$ in total), while the time required for the complete GST protocol ranged from 8 to $13 \mathrm{~min}$. Similar exercise protocols responsible for provoking reflux have been completed in $>15 \mathrm{~min},{ }^{20,37}$ and exercise duration may be an important factor in inducing GER events during these activities.

The lack of difference in the proportion of participants who experienced reflux during both baseline and all physiotherapy interventions between those prescribed anti-reflux medication and treatment naïve participants was an unexpected finding. The approach to cessation of therapy in the current study was in accordance with current rec- ommendations ${ }^{40}$ and was similar to previous reports. ${ }^{8,9}$ In addition, as a minimum of $24-48$ hours is required to restore maximal acid secretory capacity, this was considered ample washout time of medications. ${ }^{41}$ As no participant had previously completed diagnostic testing prior to commencement of anti-reflux medication, the results may imply that it may be important for some individuals with COPD or bronchiectasis to undertake objective testing for diagnostic confirmation of GER.

There are limitations to this study. Due to the invasive nature of esophageal $\mathrm{pH}$ monitoring, the inclusion of only participants with confirmed GER for the exercise and PEP therapy tasks was not possible, as a substantial proportion would have declined to participate in a repeated 24 hour study. The results may be influenced by the circadian rhythm of gastric acid secretion, with basal acid output being at its peak in the late evening hours and lowest in the early morning in healthy individuals as well as those diagnosed with GER. ${ }^{42}$ However, each physiotherapy task was completed outside of these time frames and this fluctuation is unlikely to influence the observations. In addition, this study did not explore the possible impact of GER on lung disease in COPD or bronchiectasis, a subject requiring further research.

\section{Conclusions}

In conclusion, patients with COPD and patients with bronchiectasis may experience episodes of GER during PEP therapy, the 6MWT, and the GST, suggesting that some individuals are more susceptible to reflux during specific physiotherapy tasks. However, as these activities did not increase the frequency of these incidents over BGT, the occurrence of these GER events is of little concern. Therefore, this standardized airway clearance technique and these formal measures of endurance exercise capacity and upper limb function do not require any modification to limit the risk of GER events in COPD or bronchiectasis.

\section{REFERENCES}

1. Vestbo J, Prescott E, Lange P. Association of chronic mucus hypersecretion with $\mathrm{FEV}_{1}$ decline and chronic obstructive pulmonary disease morbidity. Copenhagen City Heart Study Group. Am J Respir Crit Care Med 1996;153(5):1530-1535.

2. Koulouris N, Retsou S, Kosmas E, Dimakou K, Malagari K, Mantzikopoulos G, et al. Tidal expiratory flow limitation, dyspnoea and exercise capacity in patients with bilateral bronchiectasis. Eur Respir J 2003;21(5):743-748.

3. Ten Hacken NHT, Wijkstra PT, Kerstjens HA. Treatment of bronchiectasis in adults. BMJ 2007;335(7629):1089-1093.

4. Nici L, Donner CF, Wouters EF, Zuwallack R, Ambrosino N, Bourbeau J, et al. American Thoracic Society/European Respiratory Society statement on pulmonary rehabilitation. Am J Respir Crit Care Med 2006;173(12):1390-1413.

5. Abramson MJ, Crockett AJ, Frith PA, McDonald CF. COPDX: an update of guidelines for the management of chronic obstructive pul- 


\section{UpRIght PEP AND EXERCISE FOR REFLUX IN COPD AND BRONCHIECTASIS}

monary disease with a review of recent evidence. Med J Aust 2006; 184(7):342-345.

6. Casanova C, Baudet J, del Valle Velasco M, Martin J, Aguirre-Jaime A, Pablo de Torres J, et al. Increased gastro-oesophageal reflux disease in patients with severe COPD. Eur Respir J 2004;23(6):841845 .

7. Kempainen R, Savik K, Whelan T, Dunitz J, Herrington C, Billings $\mathrm{J}$. High prevalence of proximal and distal gastroesophageal reflux disease in advanced COPD. Chest 2007;131(6):1666-1671.

8. Sweet M, Herbella F, Leard L, Hoopes C, Golden J, Hays S, et al. The prevalence of distal and proximal gastroesophageal reflux in patients awaiting lung transplantation. Ann Surg 2006;244(4):491497.

9. Koh W, Lee J, Kwon Y, Lee K, Suh G, Chung M, et al. Prevalence of gastroesophageal reflux disease in patients with nontuberculous mycobacterial lung disease. Chest 2007;131(6):1825-1830.

10. Dent J, Holloway R, Toouli J, Dodds W. Mechanisms of lower oesophageal sphincter incompetence in patients with symptomatic gastroesophageal reflux. Gut 1988;29(8):1020-1028.

11. Schoeman MN, Tippett MD, Akkermans LM, Dent J, Holloway RH. Mechanisms of gastroesophageal reflux in ambulant healthy human subjects. Gastroenterology 1995;108(1):83-91.

12. Pandolfino J, Zhang C, Ghosh S, Han A, Boniquit C, Kahrilas P. Transient lower esophageal sphincter relaxations and reflux: mechanistic analysis using concurrent fluoroscopy and high-resolution manometry. Gastroenterology 2006;131(6):1725-1733.

13. Mittal R, Balaban D. The esophagogastric junction. N Engl J Med 1997;336(13):924-932.

14. Addington W, Stephens R, Phelipa M, Widdicombe J, Ockey R. Intra-abdominal pressures during voluntary and reflex cough. Cough 2008;4:2.

15. Button B, Heine R, Catto-Smith A, Olinsky A, Phelan P, Ditchfield $\mathrm{M}$, et al. Chest physiotherapy in infants with cystic fibrosis: to tip or not? A give-year study. Pediatr Pulmonol 2003;35(3):208-213.

16. Button B, Heine R, Catto-Smith A, Phelan P. Postural drainage in cystic fibrosis: is there a link with gastro-oesophageal reflux? J Paediatr Child Health 1998;34(4):330-334.

17. Button B, Heine R, Catto-Smith A, Phelan P, Olinsky A. Postural drainage and gastro-oesophageal reflux in infants with cystic fibrosis. Arch Dis Child 1997;76(2):148-150.

18. Falk M, Kelstrup M, Andersen JB, Falk P, Stovring S, Gothgen I. Improving the ketchup bottle method with positive expiratory pressure (PEP), in cystic fibrosis. Eur J Respir Dis 1984;65(6):423-432.

19. Lee A, Button B, Denehy L. Current Australian and New Zealand physiotherapy practice in the management of patients with bronchiectasis and COPD. NZ J Physiother 2008;32(2):49-58.

20. Ravi N, Stuart R, Byrne P, Reynolds J. Effect of physical exercise on esophageal motility in patients with esophageal disease. Dis Esophagus 2005;18(6):374-377.

21. Clark C, Kraus B, Sinclair J, Castell D. Gastroesophageal reflux induced by exercise in healthy volunteers. JAMA 1989;261(24): 3599-3601.

22. Hill C, Denehy L, Holland A, McDonald C. Measurement of functional activity in chronic obstructive pulmonary disease. J Cardiopul Rehabil Prev 2008;28(6):402-409.

23. Pauwels RA, Buist AS, Ma P, Jenkins CRR, Hurd SS; GOLD Scientific Committee. Global strategy for the diagnosis, management and prevention of chronic obstructive pulmonary disease. National Heart, Lung and Blood Institute and World Health Organisation Global Initiative for Chronic Obstructive Pulmonary Disease (GOLD): executive summary. Respir Care 2001;46(8):798-825.
24. Bhalla M, Turcios N, Aponte V, Jenkins M, Leitman B, McCauley D, et al. Cystic fibrosis: scoring system with thin-section CT. Radiology 1991;179(3):783-788.

25. Pehl C, Boccali I, Hennig M, Schepp W. pH probe positioning for 24-hour $\mathrm{pH}$-metry by manometry or $\mathrm{pH}$ step-up. Eur J Gastroenterol Hepatol 2004;16(4):375-382.

26. Fass R, Hell R, Sampliner R, Pulliam G, Graver E, Hartz V, et al. Effect of ambulatory 24-hour esophageal $\mathrm{pH}$ monitoring on refluxprovoking activities. Dig Dis Sci 1999;44(11):2263-2269.

27. Committee on Proficiency Standards for Clinical Pulmonary Function Laboratories. ATS Statement: guidelines for the six-minutewalk test. Am J Respir Crit Care Med 2002;166(1):111-117.

28. Borg G. Psychophysical bases of perceived exertion. Med Sci Sports Exerc 1982;14(5):377-381

29. Jamieson J, Stein H, DeMeester T, Bonavina L, Schwizer W, Hinder $\mathrm{RA}$, et al. Ambulatory 24-H esophageal $\mathrm{pH}$ monitoring: normal values, optimal thresholds, specificity, sensitivity and reproducibility. Am J Gastroenterol 1992;87(9):1102-1111.

30. Dobhan R, Castell D. Normal and abnormal proximal esophageal acid exposure: results of ambulatory dual-probe $\mathrm{pH}$ monitoring. Am J Gastroenterol 1993;88(1):25-29.

31. DeMeester T, Johnson L, Joseph G, Toscano M, Hall A, Skinner D. Patterns of gastroesophageal reflux in health and disease. Ann Surg 1976;184(4):459-470.

32. Ing AJ, Ngu MC, Breslin AB. Pathogenesis of chronic persistent cough associated with gastroesophageal reflux. Am J Respir Crit Care Med 1994;149(1):160-167.

33. Hodges $\mathrm{P}$, Gandevia S. Changes in intra-abdominal pressure during postural and respiratory activation of the human diaphragm. J Appl Physiol 2000;89(3):967-976.

34. Mittal RK, Rochester DF, McCallum RW. Electrical and mechanical activity in the human lower esophageal sphincter during diaphragmatic contraction. J Clin Invest 1988;81(4):1182-1189.

35. Dent J, Dodds W, Hogan W, Toouli J. Factors that influence induction of gastroesophageal reflux in normal human subjects. Dig Dis Sci 1988;33(3):270-275.

36. Pandolfino J, Bianchi L, Lee T, Hirano I, Kahrilas P. Esophagogastric junction morphology predicts susceptibility to exercise-induced reflux. Am J Gastroenterol 2004;99(8):1430-1436.

37. Maddison KJ, Shepherd KL, Hillman DR, Eastwood PR. Function of the lower esophageal sphincter during and after high-intensity exercise. Med Sci Sports Exerc 2005;37(10):1728-1733.

38. Troosters T, Vilaro J, Rabinovich R, Casas A, Barbera J, RodriguezRoisin R, et al. Physiological responses to the 6-min walk test in patients with chronic obstructive pulmonary disease. Eur Respir J 2002;20(3):564-569.

39. Cahalin L, Pappagianopopulos P, Prevost S, Wain J, Ginns L. The relationship of the 6-min walk test to maximal oxygen consumption in transplant patients with end-stage lung disease. Chest 1995;108(2): 452-459.

40. American Gastroenterology Association. Medical position statement on the use of esophageal pH recording. Gastroenterology 1996; 110(6):1981-1996.

41. Wolfe MM, Sachs G. Acid suppression: optimising therapy for gastroduodenal ulcer healing, gastro-oesophageal reflux disease and stress-related erosive syndrome. Gastroenterology 2000;118(2 Suppl 1):S9-S31.

42. Zentilin P, Dulbecco P, Bilardi C, Gambaro C, Iiritano E, Biagini R, et al. Circadian patterns of intragastric acidity in patients with nonerosive reflux disease (NERD). Ailment Pharmacol Ther 2003;17(3): 353-359. 